\title{
El Estado y el capital financiero en Argentina ENTRE 2002 Y 2012. LA DEUDA PÚBLICA
}

\author{
Marisa Bordón ${ }^{1}$
}

Fecha de recepción: 27 de octubre de 2016. Fecha de aceptación: 08 de marzo de 2017.

\section{RESUMEN}

Con el fin de encontrar indicios acerca de la posición del capital financiero en la dinámica económica argentina entre 2002 y 2012, se han analizado algunos aspectos de su relación con el Estado. El "desendeudamiento" llevó a la desarticulación parcial de la deuda pública como eje de la actividad especulativa, pero conservó su rentabilidad y posición prioritaria en las decisiones de política económica. Por otro lado, la regulación financiera contribuyó a conservar el mecanismo de la deuda y no alteró significativamente la posición central del capital financiero.

Palabras clave: política económica, deuda pública, capital financiero, Estado, desendeudamiento, regulación financiera.

Clasificación JEL: G18, G28, H63, O18, R11.

\section{The State and Financial Capital in Argentina Between 2002 AND 2012. Public Debt}

\begin{abstract}
In order to elucidate the role of financial capital in the dynamics of the Argentine economy between 2002 and 2012, this paper analyzes several aspects of the relationship between financial capital and the State. "Debt reduction" partially dismantled public debt as the backbone of speculative activity, although it maintained its profitability and priority position in economic policy decision-making. On the other hand, financial regulation contributed to keeping the mechanism of debt and did not significantly alter the central role played by financial capital.
\end{abstract}

Key Words: Economic policy, public debt, financial capital, the State, debt reduction, financial regulation.

${ }^{1}$ Universidad de Zaragoza, España. Correo electrónico: bordon_marisa@hotmail.com 
L'ÉTAT ET LE CAPITAL FINANCIER EN ARGENTINE ENTRE 2002 ET 2012. LA DETTE PUBLIQUE

\section{Résumé}

Afin de trouver des indices sur la position du capital financier dans la dynamique économique entre les années 2002 et 2012, on a analysé quelques aspects de son rapport avec l'État. Le " désendettement " a conduit au démantèlement partiel de la dette publique comme moteur de l'activité de spéculation, mais a conservé sa rentabilité et sa position prioritaire dans les décisions de politique économique. D’un autre côté, la réglementation financière a contribué à la conservation du mécanisme de la dette et elle n'a pas affecté de manière considérable la position centrale du capital financier. Mots clés: politique économique, dette publique, capital financier, État, désendettement, réglementation financière.

\section{A CAPITAL DO ESTADO E DAS FINANÇAS NA ARGENTINA ENTRE 2002 E 2012. A DÍVIDA PÚBLICA \\ Resumo}

A fim de encontrar pistas sobre a posição do capital financeiro na dinâmica economia argentina entre 2002 e 2012, foram analisados alguns aspectos da sua relação com o Estado. O "desendividamento" levou ao desmantelamento parcial da dívida pública como eixo da atividade especulativa, mas manteve a sua rentabilidade e sua posiçáo prioritária nas decisóes de política econômica. Por outro lado, a regulamentação financeira contribuiu para preservar o mecanismo da dívida e não alterou significativamente a posição central do capital financeiro.

Palavras-chave: política económica, dívida pública, capital financeiro, Estado, regulação financeira.

2002和2012年阿根廷国家和金融资本：公共债务

玛丽莎・博尔登

摘要

为找到2002年和2012年金融资本在阿根廷经济运行中的地位，本文分析 了金融资本与国家相关的几个方面。去杠杆化导致了公共债务作为投机活 动轴心的分化, 但是保留了公共债务的收益及其在经济政策中的优先地 位。另一方面, 金融监管促进了债务机制的保留, 且并为显著改变金融资 本的中心地位。

关键词：经济政策、公共债务、金融资本、国家、去杠杆化、金融监管 


\section{INTRODUCCIÓN}

En la década de los noventa, el Estado argentino cumplió un papel fundamental generando las condiciones que permitieron que el capital financiero se ubicara en el centro de la escena económica. El pago de la deuda era financiado con más deuda debido a los desequilibrios fiscales y a la cuenta corriente que la propia política económica profundizaba (sobrevaluación de la moneda y requerimientos de la base monetaria). A través de este mecanismo, los capitales entraban a la economía, se valorizaban en el mercado financiero gracias al diferencial de tipos de interés, para luego fugarse al exterior por las facilidades de la liberalización financiera y la convertibilidad (Basualdo, 2006). La necesidad de financiación y las políticas monetaria y cambiaria elevaban la rentabilidad de la deuda pública ante la necesidad de incrementar su atractivo y compensar el riesgo. Las políticas monetaria y cambiaria, en este sentido, fueron canales de influencia directa sobre el rol preponderante de las finanzas en el país durante el periodo de convertibilidad.

La insostenibilidad del modelo económico llevó a la crisis que estalló en 2001, cuyos efectos fueron devastadores en el país, las condiciones sociales de la población sufrieron un deterioro inédito, llevando las tasas de desempleo a niveles superiores al 20\% y el índice de pobreza en torno al $40 \%$. Pese al déficit comercial y fiscal, la imposibilidad de acceder a la financiación internacional, la interrupción de la línea de pagos y cobros, y el estallido social, la economía argentina se recuperó en un tiempo récord, consiguió sanear las cuentas públicas y externas, y presentó elevadas tasas de crecimiento en casi todo el periodo. Este desempeño fue propiciado, en cierta medida, por una orientación diferente de su política económica, sin embargo, no indica per se un cambio en la posición del capital financiero.

El presente texto busca indagar sobre la existencia de cambio o continuidad en la posición del capital financiero en la economía argentina desde el análisis de su relación con el Estado en el periodo 2002-2012. ${ }^{2}$ Para ello, se abordó el análisis de los dos elementos que resultaban determinantes para el rol del capital financiero en el periodo anterior. El primer apartado está dedicado al análisis de la evolución de la deuda pública y los matices del alcance de la política de "desendeudamiento", y el segundo apartado, a los efectos de la regulación financiera, que como se verá guarda estrecha relación con las características de este endeudamiento.

Para una revisión más amplia del capital financiero se recomienda consultar Golla (2006), Cobe (2009), Kupelian \& Rivas (2011) y Golonbek \& Mareso (2011). En estos trabajos se abordan los cambios que se producen en el sistema financiero durante los años de la posconvertibilidad. 


\section{EL ALCANCE DE LA POLÍTICA DE “DESENDEUDAMIENTO”}

A mediados de 2000 el oficialismo comenzó a hablar de un proceso de "desendeudamiento" en Argentina. ${ }^{3}$ Esta afirmación se justificó, en el caso de la reestructuración, por los efectos de la quita sobre el stock de deuda defaulteada, los nuevos tipos negociados y su impacto en la sostenibilidad en los pagos. El principal indicador de sostenibilidad, el porcentaje del stock de deuda en el producto interno bruto (PIB), se redujo considerablemente desde el primer canje en 2005 (CENDA, 2010).

Por otro lado, la no intervención del Fondo Monetario Internacional (FMI) en este proceso y el pago de la deuda con este organismo internacional también formaron parte del argumento, puesto que con ello se reducía la deuda externa y se ganaba soberanía sobre la política económica. Ambas cuestiones habrían llevado al fin de la dependencia financiera internacional.

No obstante, surgieron algunos trabajos que cuestionaron la existencia de una verdadera reducción de la carga para el Estado. Es desde esta visión crítica que se matizará el alcance de la gestión de la deuda en la desarticulación del mecanismo existente en el periodo anterior y sus implicaciones en la posición del capital financiero. ${ }^{4}$ En primer lugar, se analizará la evolución de los indicadores sobre los que se sostiene la tesis del "desendeudamiento" y, en segundo lugar, se verán los argumentos sobre los que se asienta la crítica de dicha tesis, relacionados con el stock de deuda, los mecanismos de la reestructuración y el reemplazo de deuda externa por interna.

\subsection{Indicadores del "desendeudamiento" y el crecimiento de la deuda pública}

Respecto a la evolución de los indicadores del "desendeudamiento" se observa en la gráfica 1 que el stock de deuda pública creció significativamente durante el periodo en el que se mantuvo la suspensión de pagos (2002-2004), no sólo por los efectos de la crisis y la devaluación, sino también por la intervención del Estado, especialmente en el sector financiero. El canje supuso una reducción significativa de la deuda que pasó de 191.296 a 128.630 millones de dólares en 2005.

Sobre la tesis del "desendeudamiento" véase CENDA (2010), Kulfas (2014) y Nemińa (2012).

Es pertinente aclarar que no está dentro de los objetivos de este trabajo adentrar en el debate del "desendeudamiento", describiendo las diferentes posturas y sus matices. 
Fue a partir de entonces que siguió una tendencia creciente hasta llegar a los 197.464 millones de dólares en 2012. Sin embargo, la tesis de "desendeudamiento" no tuvo en cuenta este crecimiento, sino la sostenibilidad de la deuda, es decir, lo gestionable que resultaba el pago de sus servicios con recursos propios.

El stock de deuda sobre el PIB, el stock de deuda externa sobre el PIB, el porcentaje sobre el pIB de los intereses y del servicio de la deuda han sido los principales indicadores de la salud de dicha gestión.

El stock de deuda como porcentaje del PIB alcanzó su máximo nivel en 2002, 166.4\%, por el efecto que tuvo la devaluación sobre el stock de deuda, ya que casi el $80 \%$ se hallaba denominado en dólares. También incrementaron la deuda pública el rescate a la banca y demás medidas que se implementaron para enfrentar la crisis. No obstante, debe tenerse en cuenta el efecto que la fuerte caída del pIB tuvo en el denominador de este indicador como consecuencia de la recesión económica de aquel año.

En la gráfica 1 se observa que el stock de deuda en el pIB desciende a partir de 2002, presenta una caída muy significativa luego de la reestructuración, pasando de $127.3 \%$ en 2004 a $73.9 \%$ en 2005 , para acabar el periodo en

Gráica 1. Deuda pública (en millones de dólares) e indicadores de sostenibilidad, 1998-2012

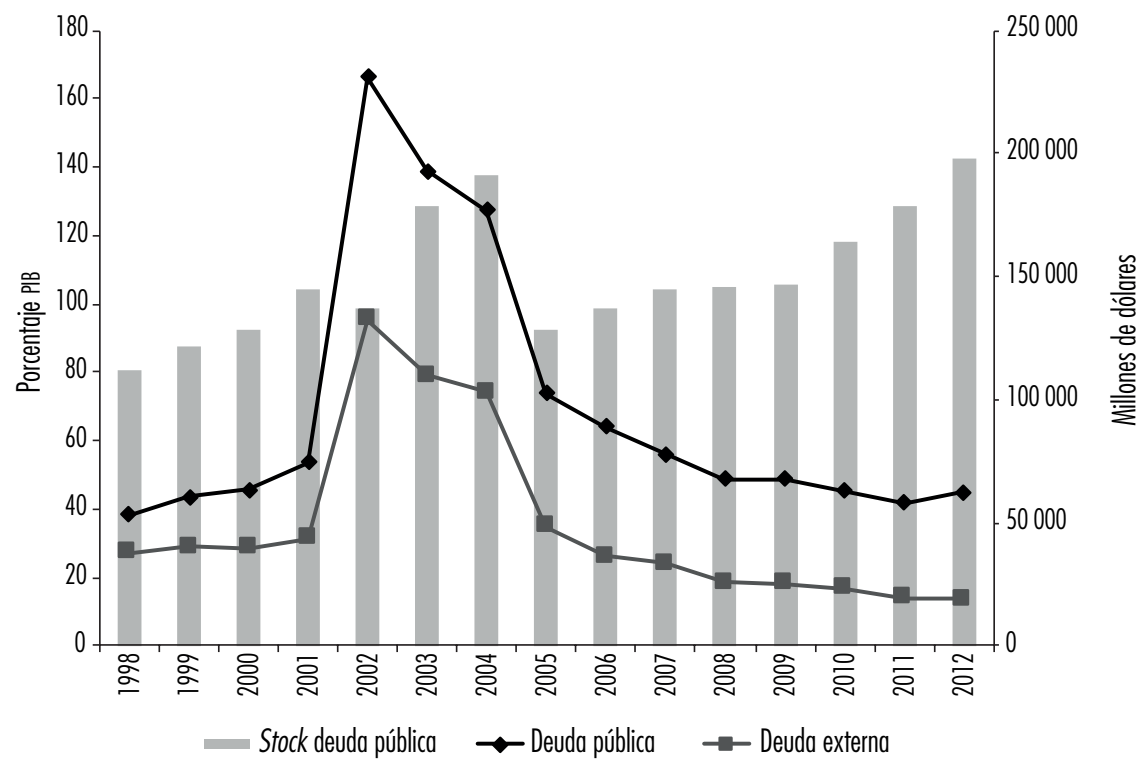

Fuente: Secretaría de Finanzas del Ministerio de Economía. 
un $44.9 \%$. Aunque la caída de este indicador es relevante, especialmente si tenemos en cuenta los niveles presentes en los años que dura el default, debe matizarse que sus valores no llegan a ser menores que los presentes en 1998 (38.2\%), momento en el que la deuda se encontraba en el centro de la economía como eje dinamizador de la actividad especulativa y como sostén del modelo macroeconómico.

Más significativo resulta el caso del segundo indicador: el stock de deuda externa sobre el PIB. Este ratio pasó de 31.5\% del PIB en 2001 a 24.1\% del PIB en 2007, con una reducción significativa en 2012, cuando su porcentaje llegó al $13.7 \%$ del piв. Como porcentaje de la deuda bruta total, la deuda en moneda extranjera pasó de $96.9 \%$ en 2001 a 52.8\% en 2007. En 2012 este porcentaje aumentó ligeramente a 59\%. Este indicador parece mostrar que, efectivamente, existe una menor exposición al endeudamiento externo en este periodo.

Ambos indicadores muestran un mejor desempeño en términos de gestión, en el caso del endeudamiento externo mucho más claro. Sin embargo, el stock de deuda pública nacional siguió creciendo, incluso en el periodo de superávit fiscal financiero entre 2005 y 2008, hecho que debería haber verificado una reducción del endeudamiento neto.

Se abrió entonces el debate sobre el éxito de la reestructuración y el fin del problema de la deuda externa. Sus detractores tradujeron el concepto de "desendeudamiento" en un reemplazo de deuda externa por interna, catalogando la política del gobierno al respecto como una excusa para seguir pagando rigurosa y puntualmente una deuda de cuestionable legitimidad ${ }^{5}$ (Damill et al., 2005; Giuliano, 2006; Lo Vuolo \& Seppi, 2008).

\subsection{Las críticas a la tesis del "desendeudamiento"}

Los argumentos utilizados por los autores que denunciaron "la falacia del desendeudamiento" podrían agruparse en tres: el impacto de los nuevos mecanismos impuestos por la reestructuración en el stock de deuda, el impacto de estos mecanismos en la carga que supone el pago de sus intereses y el reemplazo de deuda externa por interna.

Los nuevos mecanismos sobre los que se realizó la reestructuración de la deuda en suspensión de pago han sido: el ajuste por el Coeficiente de Estabilización de Referencia (CER) de los bonos Cuasi Par, los intereses capitalizables y los cupones vinculados al PIB.

5 Para más información sobre la legitimidad de la deuda pública véase Basualdo (2006). 
Cuadro 1. Intereses capitalizados ${ }^{\star}$ y cupones ligados al PIB**, millones de dólares

\begin{tabular}{lcc}
\cline { 2 - 3 } & Cupones ligados al PIB & Intereses capitalizados \\
\hline 2006 & 389 & 969 \\
2007 & 821 & 990 \\
2008 & 1302 & 1034 \\
2009 & 1417 & 1033 \\
2010 & & 1761 \\
2011 & 2487 & 1273 \\
2012 & 3520 & 1030 \\
\hline Total & 9936 & 8090 \\
\hline
\end{tabular}

* Los datos referentes a intereses capitalizados incluyen además de los generados por los bonos del canie, los bonos consolidación (BOCON). ** Los datos de la columna de cupones ligados al PIB son los generados por el crecimiento de la economía el año anterior.

Fuente: elaboración propia con base en datos del Boletín Fiscal (Secretaría de Hacienda), p. 12, 26 de marzo de 2011, Instituto Argentino de Mercado de Capitales (IAMC) y BCRA.

Tanto el ajuste por CER como los intereses capitalizados incrementaron el stock de deuda con el paso del tiempo. En el primer caso, el impacto ocasionado por la inflación no se computa hasta que, efectivamente, se procede al incremento de la deuda. Entre 2006 y 2008 se verificó que el ajuste por CER incrementó el stock de deuda en 12.731 millones de dólares. ${ }^{6}$

Desde los enfoques críticos se ha denunciado que la importancia de la indexación de la deuda en el coste de la misma fue la razón del cambio de metodología utilizada por el INDEC en el cálculo del IPC a partir de 2007. La nueva metodología habría subestimado la subida generalizada de los precios, llevando a la pérdida de credibilidad en la fiabilidad de los datos aportados por este organismo (Lo Vuolo \& Seppi, 2008).

Luego de la estatización de las Administradoras de Jubilaciones y Pensiones (AFJP) en 2008, la mayor parte de esa deuda quedó en manos de la Administración Nacional de la Seguridad Social (ANSES). Su rentabilidad favoreció al fondo aportado para el sistema previsional, pero el ajuste por un Índice del

6

Datos del Boletín Fiscal (trimestral) del Ministerio de Economía y Producción. Secretaría de Hacienda. 
Precio al Consumidor (IPC) menor al realmente existente licuó parte de estos beneficios (Giuliano, 2013). Todo lo contrario, ocurría durante los años 2005, 2006 y 2007, cuando el ajuste por CER se convirtió en uno de los mecanismos que impulsó la rentabilidad de su principal poseedor: el sistema financiero local.

Según los datos del cuadro 1, los intereses capitalizados aumentaron la deuda en 8.090 millones de dólares entre 2006 y 2012. Su impacto es relevante, puesto que los intereses a pagar se fueron calculando sobre una base cada vez más grande, lo que implicó, en última instancia, un pago mayor.

Además, en valores constantes, los intereses capitalizados en el periodo 2002-2006 representaron casi el 40\% del total de intereses; el promedio anual de la suma entre intereses pagados y capitalizados se aproxima al valor de intereses pagados en 1999 y supera al resto de los años de la década de los noventa (Lo Vuolo \& Seppi, 2008, p. 15).

Otro de los elementos que se pone en cuestión a la hora de analizar el stock de deuda, es la exclusión de la deuda holdout; aquella parte de la deuda que no fue canjeada en 2005 ni en 2010. Al eliminarse la "cláusula cerrojo" y abrirse el canje en 2010, la deuda holdout se convirtió en deuda potencial en lugar de deuda no reconocida (Giuliano, 2013). Esta deuda representaba en 2012 un importe de 11.482 millones de dólares (capital e intereses, sin los intereses moratorios ni punitorios).

El segundo argumento en contra de la tesis del "desendeudamiento" está relacionado con el peso del pago de intereses y servicios. En el pasado, el enorme peso de los servicios de la deuda impactaba en el déficit público y, con ello, en el aumento del endeudamiento para su pago. Este mayor endeudamiento llevaba, a su vez, a que las nuevas emisiones de deuda necesitaran ser cada vez más atractivas, es decir, que devengaran un mayor interés y, por lo tanto, resultaran más rentables, aunque esto significara un mayor coste para la economía argentina.

El canje habría significado una reducción en el peso de los intereses, convirtiendo el problema de la deuda en un problema gestionable. Sin embargo, tanto la quita como la reducción de intereses fueron compensadas con los mecanismos de la reestructuración que conservaron su rentabilidad y, por lo tanto, mantuvieron su elevado coste (Damill et al., 2005; Giuliano, 2006; Lo Vuolo y Seppi, 2008; Capello \& Grión, 2010).

A simple vista, los intereses a pagar se redujeron como resultado de la reestructuración. Mientras que en diciembre de 2001 equivalían a 10.175 millones de dólares anuales, en 2005 presentaban un valor cercano a los 3.205 millones de dólares (Lo Vuolo \& Seppi, 2008). 
Sin embargo, en la negociación de la deuda se otorgaron ciertos privilegios a los proveedores que ingresaron al canje. Entre ellos, la fecha de emisión de los bonos el 31 de diciembre de 2003, por lo que, a pesar de haberse realizado el canje en 2005, los intereses comenzaron a devengarse un año antes.

En los datos que nos muestra la gráfica 2 (eje principal) vemos cómo el porcentaje de los servicios de la deuda en el рів cae de 15.3\% en 2001 a $10.2 \%$ en $2007 .^{7}$ Pese a ello, si tenemos en cuenta los datos de los últimos ańos de la década anterior, continuaron representando un porcentaje similar.

Algo parecido ocurrió con los intereses. En 2001 representaban el 3.8\% del PIB y se redujeron a $1.9 \%$ en 2005 . Y, aunque desde entonces se mantuvieron en torno a ese porcentaje, en 1994 se encontraban en el 1.2\% del pib. Es decir, la carga en términos de coste de la deuda parecería reducirse significativamente, si se tienen en cuenta los niveles correspondientes al periodo de crisis únicamente.

Con el fin de poder matizar los datos que se ofrecen sobre la reducción del peso de la deuda como resultado de la gestión en este periodo, se han incluido en la gráfica los datos de los intereses y servicios en relación con el pIB desde 1993. De esta forma, puede apreciarse que existe una reducción, pero que apenas se acerca a los niveles de principios de la década de los noventa.

La gráfica 2 (eje secundario) también recoge el porcentaje de los intereses y del servicio de la deuda en relación con los recursos tributarios. En ambos casos se redujo en más del 50\%, teniendo en cuenta el peso que representaban en el 2000. Esta reducción muestra una significativa mejora en la sostenibilidad del endeudamiento, dado el incremento de los ingresos fiscales durante todo este periodo, no sólo por retenciones a la exportación, sino también como resultado del aumento de la recaudación por las elevadas tasas de crecimiento de la economía.

Sin embargo, si tomamos como indicador el peso de los intereses en el gasto corriente, recogido en la gráfica 3, observamos que entre 1996 y 1998 presentaba un nivel similar al del periodo 2005-2007, siendo en promedio el $13 \%$ de estos gastos. Este promedio se mantuvo también entre 2008 y 2012; la reestructuración no parece haber aligerado el peso de la deuda pública en el gasto público.

Desde la tesis del "desendeudamiento", algunos autores matizan el impacto de la reducción del peso de los intereses, reconociendo que el pago de la deuda llevó a la erosión de la salud de las cuentas públicas a partir de 2007 (CENDA, 2010). 


\section{Marisa Bordón}

Gráfica 2. Intereses y servicios de la deuda pública como porcentaje del PIB y como porcentaje de los recursos tributarios (R.T.), 2000-2012

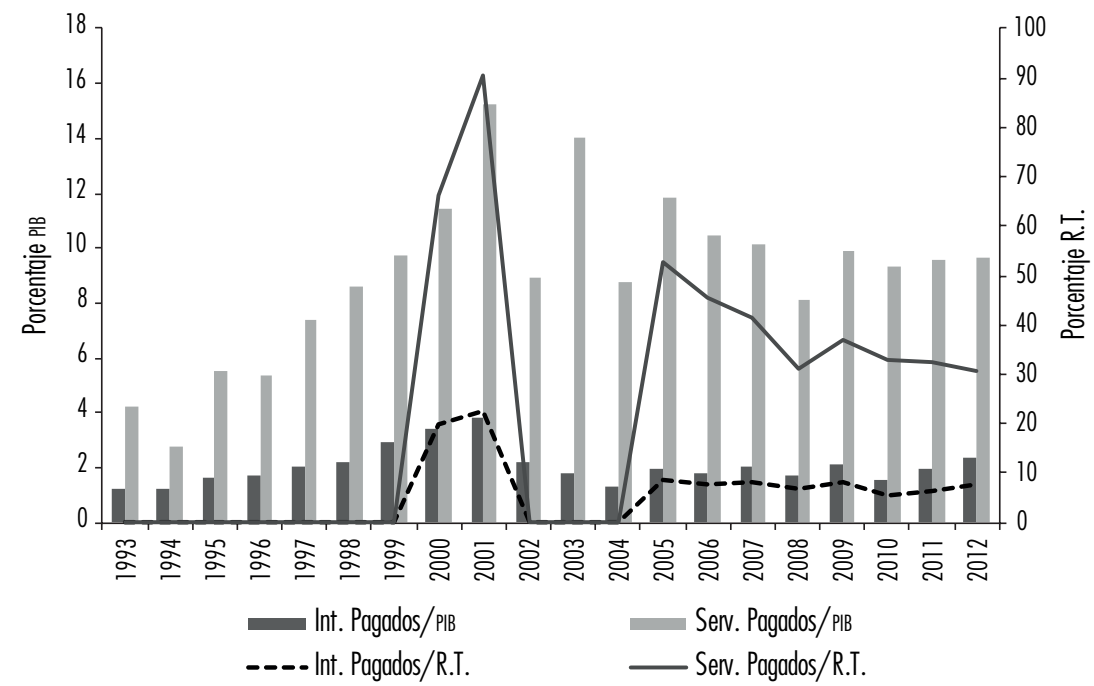

Fuente: elaboración propia con base en datos de indicadores de sostenibilidad publicados por la Secretaría de Finanzas (MECON). Los datos de intereses y servicios sobre el PIB entre 1993-1999 y 2002-2004 han sido extraídos de "Los resultados de la política de desendeudamiento" de Marcelo Capello y Néstor Grión.

Gráfica 3. Intereses pagados como porcentaje del gasto público, 1990-2012

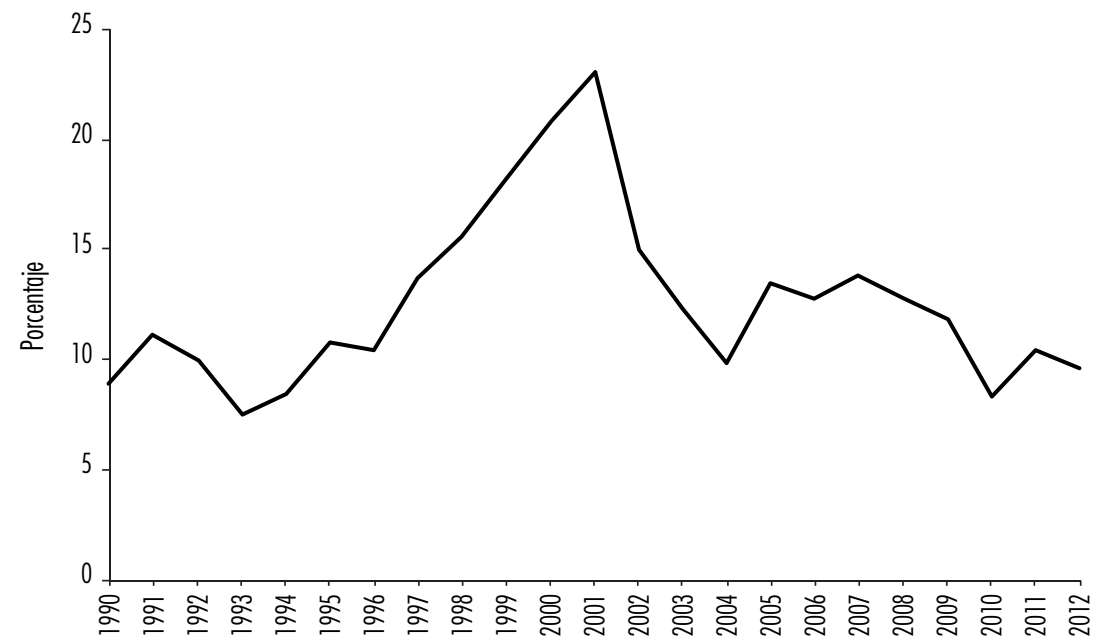

Fuente: elaboración propia con base en datos de las Operaciones del Gobierno de la Cepal (CEPALSTAT). 
Además de los intereses y servicios, el coste de la deuda también ha incluido los cupones vinculados al PIB. Estos cupones han supuesto pagos ciertos durante casi todos los ańos desde la realización del primer canje, siendo el total pagado hasta 2012 de 9.926 millones de dólares, tal como puede observarse en el cuadro 1. El cupón vinculado al PIB no computa como deuda cierta, sino que es considerado deuda contingente, aunque se hayan realizado los pagos. Por otro lado, su pago depende de la tasa de crecimiento acumulado y no sólo de la correspondiente al periodo en cuestión; puede pagarse incluso si la economía crece menos que lo proyectado por el gobierno (Lo Vuolo \& Seppi, 2008).

En el cuadro 2 se calcula el coste implícito de la deuda teniendo en cuenta los incrementos del ajuste por CER y los pagos de los cupones del PIB hasta 2007. Vemos que en 1998 y 1999 (6.02 y 6.68\%) no difiere en gran medida del presente en 2006 y 2007 (6.71\% ambos ańos).

Respecto a los mecanismos implementados en la reestructuración y su significado en términos de rentabilidad, Lo Vuolo \& Seppi (2008, p. 25) dicen que "los papeles de la deuda siguen dando un rendimiento muy elevado financieramente, por lo que la deuda pública argentina sigue siendo un elemento importante de canalización de los ahorros líquidos y de valorización del capital financiero". Sólo que la rentabilidad actual se vincula a los de capitalización de intereses y de ajuste del capital principal en lugar de a los intereses pagados.

Cuadro 2. Coste implíito de la deuda del sector público nacional (porcentaje)

\begin{tabular}{lcccccccccc}
\hline & 1998 & 1999 & 2000 & 2001 & 2002 & 2003 & 2004 & 2005 & 2006 & 2007 \\
\hline Intereses & 6.00 & 6.60 & 7.60 & 8.30 & 1.40 & 1.60 & 1.10 & 2.80 & 2.90 & 3.10 \\
Comisiones & 0.02 & 0.08 & 0.41 & 0.83 & 0.01 & 0.03 & 0.01 & 0.02 & 0.01 & 0.01 \\
*Coste implíito & 6.02 & 6.68 & 8.01 & 9.13 & 1.41 & 1.63 & 1.11 & 2.82 & 2.91 & 3.11 \\
antes del CER y CPIB & & & & & & & & & & \\
CER Neto & 0.00 & 0.00 & 0.00 & 0.00 & 6.70 & 0.00 & 1.10 & 4.60 & 3.20 & 2.70 \\
Cupón PIB & 0.00 & 0.00 & 0.00 & 0.00 & 0.00 & 0.00 & 0.00 & 0.30 & 0.60 & 0.90 \\
Coste implíito & 6.02 & 6.68 & 8.01 & 9.13 & 8.11 & 1.63 & 2.21 & 7.72 & 6.71 & 6.71 \\
\hline
\end{tabular}

* El coste implícito antes del CER y Cupón vinculado al PIB (CPIB) se calcula como los gastos presupuestarios en servicios de la deuda sobre el total de deuda.

Fuente: Informe Especial de Estudio de la Deuda Pública de la Auditoría General de la Nación. 
Estos instrumentos impactaron en el coste y, por tanto, en la rentabilidad que recibieron los acreedores que entraron al canje. Se negociaron menores intereses a pagar y alargamiento de plazos de pago, a cambio de incluir elementos supuestamente aleatorios.

Sin embargo, a pesar de conservar su rentabilidad y de los límites que puedan encontrarse al "desendeudamiento", hay un elemento indiscutible que supone una diferencia en la gestión del endeudamiento: la reducción del porcentaje de deuda extranjera. Esto ha sido, sin duda, una consecuencia de la reestructuración. Asimismo, ha supuesto el aumento de las obligaciones del Estado con el entramado de administraciones públicas. Se llega así al tercer argumento planteado por la corriente crítica de la gestión de la deuda pública en este periodo.

Las críticas al reemplazo de deuda externa por interna se dividen en dos. Por un lado, los cuestionamientos sobre el verdadero alcance de la "independencia" lograda respecto al capital extranjero; por el otro, las consecuencias que puede tener en el entramado de instituciones públicas financiar de forma creciente al Estado Nacional.

En 2005 se anunciaba la cancelación de la deuda con el FMI, cuya principal consecuencia sería, según quienes llevaban adelante la negociación, el fin de los condicionamientos externos a la política económica del país (Peralta Ramos, 2007). Si bien se evitaron los condicionamientos del FMI y se ganaron márgenes de libertad muy significativos, se mantuvieron ciertos condicionamientos por las cláusulas de la reestructuración ${ }^{8}$ y por la deuda que aún se mantenía con el Banco Mundial y el Banco Interamericano de Desarrollo, cuyos requisitos eran análogos a los del FMI (Giuliano, 2006). Por otro lado, la cancelación de la deuda con el FMI no implicó "desendeudamiento" puesto que, en contrapartida, se asumió una deuda con el Banco Central de la República Argentina (BCRA). ${ }^{\text {? }}$

La reducción del endeudamiento en el total del stock de deuda pública puede interpretarse como una mayor independencia financiera del Estado en cuanto a los capitales internacionales. Sin embargo, la solvencia futura de las instituciones públicas, que reemplazaron a la financiación externa, quedó comprometida por el propio modelo al erosionarse el estado de las cuentas públicas, las que comenzaron a ser deficitarias desde 2009, aunque el resultado

\footnotetext{
Para el detalle de estas cláusulas véase Giuliano (2006).

Nemiña (2012) minimiza la importancia de esta deuda interna, sostiene que la cancelación de la deuda con FMI permitió un crecimiento basado en la promoción del mercado interno y evitó hacer mayores concesiones a los acreedores externos.
} 
primario presentó cifras negativas recién a partir de 2011. No hubo pagos netos de capital; la totalidad de vencimientos por este concepto se cubrieron con emisión de nueva deuda, en su mayoría, con organismos públicos, mientras que los intereses se pagaron como parte del gasto público corriente, excluyendo los cupones vinculados al PIB, considerados como "deuda contingente" (Giuliano, 2013).

La gráfica 4 nos muestra que, a partir de 2011, el endeudamiento con el sector público representó más de la mitad de la deuda pública oficial del Estado Central.

El BCRA encabeza la lista de acreedores públicos con una deuda que en 2012 alcanzaba los 62.365 millones de dólares. ${ }^{10}$ Estas obligaciones corresponden a préstamos de reservas internacionales para el pago de deuda externa, contra la entrega de letras intransferibles, y adelantos transitorios. Luego, encontramos como fuente de la financiación pública a la ANSEs, la Administración Federal de Ingresos Públicos (AFIP), el Programa de Atención Médica Integral (PAMI) y empresas del Estado. El Banco Nación, por otra parte, mantuvo el 40\% de su cartera de crédito destinado a la financiación del Estado (Giuliano, 2013).

Gráfica 4. Deuda pública con agencias del sector público, 2009-2012

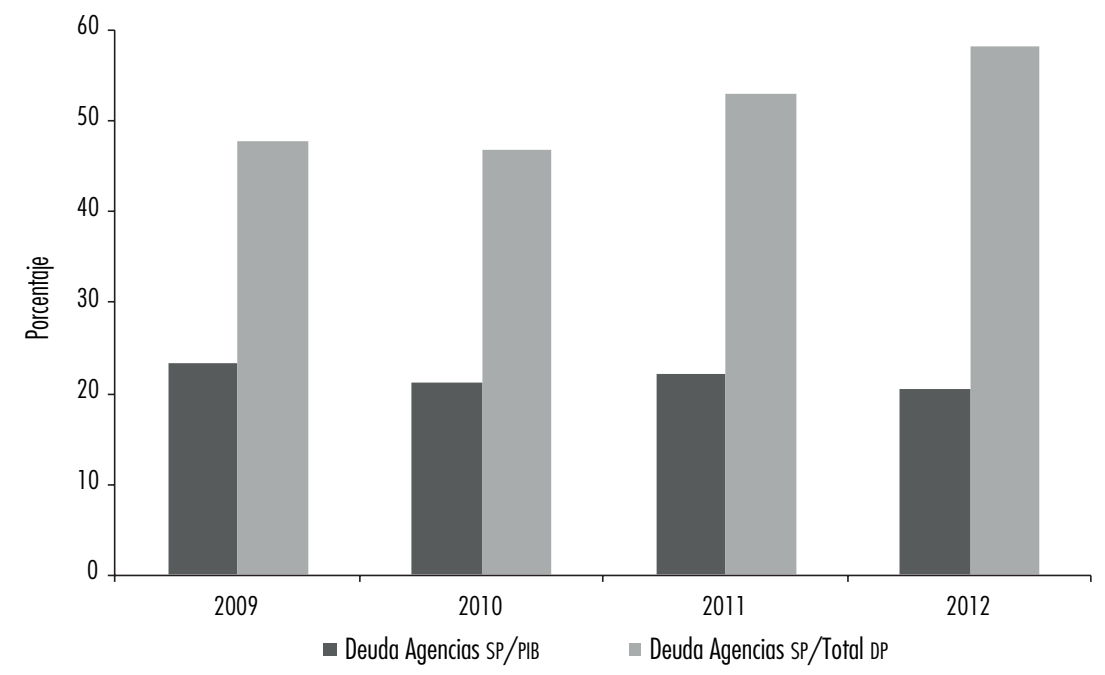

Fuente: elaboración propia con base en datos del Informe de Deuda del Banco Central (BCRA).

10

Según datos de los Estados Contables del BCra de 2013, calculado al tipo de cambio del 31 de diciembre de 2012. 
En la gráfica 5 puede verificarse que los adelantos transitorios del BCRA aumentaron su importancia en el endeudamiento público, pasando de $0.7 \%$ del total en 2002 a $8.7 \%$ en 2011 . El salto al $13.2 \%$ en 2012 coincide con una caída de las reservas internacionales. ${ }^{11}$

Por otra parte, la emisión de Notas del Banco Central (NOBAC) y Letras del Banco Central (LEBAC) fue creciente en todo el periodo. En 2012 representó el $47 \%$ del total de las reservas del BCRA, como puede observarse en los datos de la gráfica 6. La solvencia de la entidad se ve comprometida por su intervención en el sostenimiento del modelo macroeconómico (a través de la emisión de estos títulos para conservar la paridad competitiva) y la financiación al Tesoro. Esta financiación es la razón por la que el BCRA transformó lo que en un comienzo fue una política de acumulación de reservas en una de reposición de reservas. La compra de divisas, que incrementó la emisión de NOBAC y LEBAC, supuso una garantía para que el Estado pudiera pagar, a su vez, la deuda con acreedores internacionales.

En el caso del ANSES, en noviembre de 2011, el 57.6\% de la cartera del Fondo de Garantía de Sustentabilidad correspondía a títulos públicos nacionales, porcentaje que se elevó a $61.7 \%$ en el cuarto trimestre de $2012 .{ }^{12} \mathrm{El}$ traspaso desde las AFjP en 2008 no significó un cambio en la colocación de los fondos que conformaban el conjunto de aportes, su destino siguió siendo mayoritariamente la financiación pública.

Este entramado representa el sostenimiento institucional del sistema de deuda pública, cuyo coste de oportunidad fue el desvío de fondos a posibles destinos específicos por parte de los organismos públicos, relacionados con las necesidades sociales y el desarrollo (Giuliano, 2013). En su lugar, permaneció financiando un mecanismo de pagos y sosteniendo el modelo de paridad competitiva con el dólar.

Con el correr del tiempo, la necesidad de recursos, desde las administraciones públicas, para el mantenimiento de los pagos a los acreedores de la reestructuración fue cobrando importancia haciendo, una vez más, que el endeudamiento público se convirtiera en un condicionante de la política económica argentina, más allá de la proclamada independencia de los mercados internacionales de capital.

Datos del Informe Mensual (2011) y Trimestral (2012) del Fondo de Garantía de Sustentabilidad (FGS) publicado por el ANSES. El FGS está formado por los fondos que eran administrados por las AFJP. 
Gráfica 5. Porcentaje de adelantos transitorios del BCRA en la deuda pública del Estado, 2002-2012

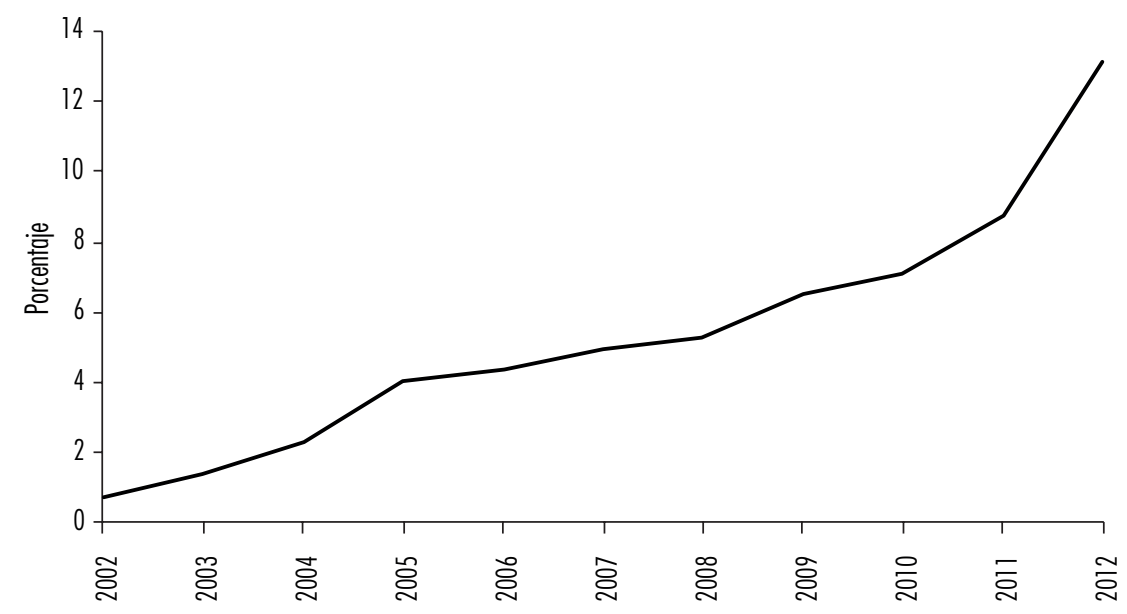

Fuente: elaboración propia con base en datos de la Secretaría de Finanzas.

Gráfica 6. Porcentaje de NOBAC y LEBAC en el total de reservas internacionales del BCRA, 2002-2012

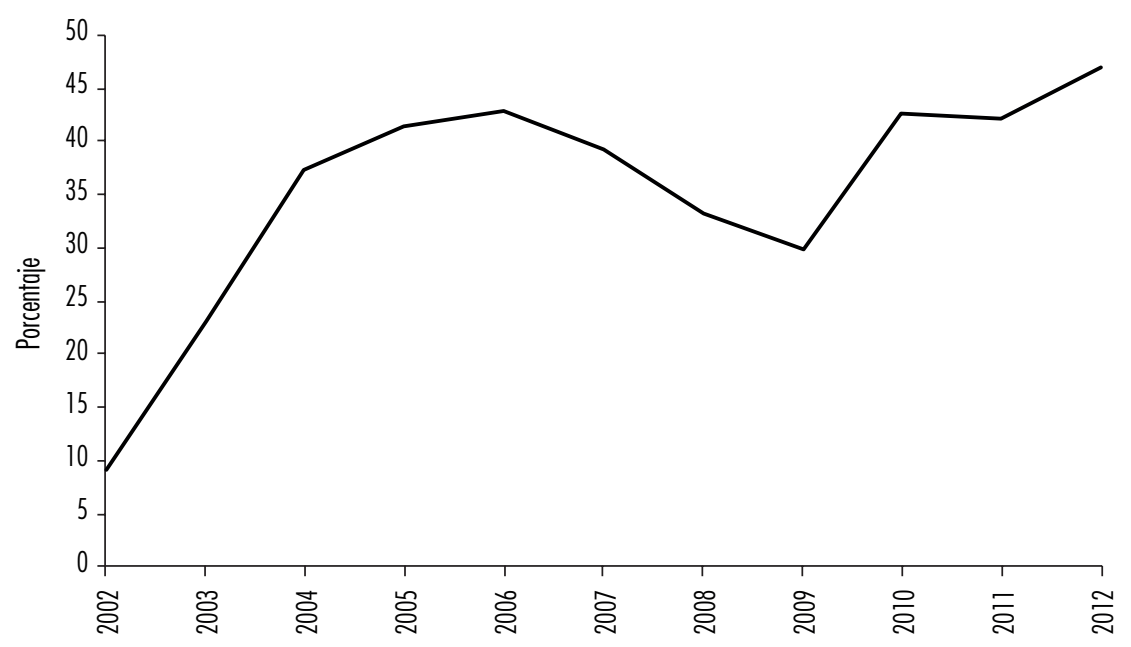

Fuente: elaboración propia con base en datos del Balance Anual-Serie semanal del BCRA. 
Por esa razón, el reemplazo de financiación externa por interna y el devenir de las medidas de política económica han llevado a algunos autores a criticar la "vocación de pago" del gobierno (Giuliano, 2013; Lo Vuolo y Seppi, 2008). Giuliano (2013) sostiene que la vocación pagadora del gobierno estaba vinculada a la necesidad de volver a los mercados internacionales de deuda, señalando como principal consecuencia la "descapitalización del BCRA" y la "desfinanciación del ANSES". Esta cuestión se puso de manifiesto posteriormente, con una tímida vuelta al endeudamiento externo, motivo por el que se realizaron ciertas negociaciones. ${ }^{13}$

Por otro lado, el pago riguroso de las obligaciones era necesario para el mantenimiento del modelo. Un aumento de las obligaciones con el exterior incrementaba el peso de los intereses en las cuentas fiscales, limitando los recursos disponibles para la aplicación de las políticas intervencionistas que han caracterizado este periodo.

La reestructuración y cancelación de la deuda con el FMI hicieron del endeudamiento interno público un factor determinante en la dinámica económica del periodo kirchnerista. La deuda externa se redujo como porcentaje de la deuda total, no por una caída del endeudamiento en general, sino por el aumento del endeudamiento interno.

Como consecuencia de la financiación pública interna para el pago de deuda externa, el superávit comercial fue sometido a una gran presión para mantener el nivel de reservas internacionales. Las divisas resultaban necesarias, tanto para el control del tipo de cambio como para el pago de las obligaciones del Estado.

Más allá de que lo aquí expuesto, ponga en duda la veracidad de la tesis del "desendeudamiento", lo significativo para el análisis es que pone en entredicho la total desaparición del mecanismo de la deuda pública como impulsor de la rentabilidad financiera y como elemento condicionante del curso de la economía argentina. El hecho de que su rentabilidad (y por tanto su coste) se mantenga en niveles similares a los presentes en la época de la "valorización financiera" ${ }^{\text {"14 }}$ (véase cuadro 2), así como la continuidad de su posición prioritaria en términos de política económica, son evidencias de que la deuda pública conservó parte del papel que protagonizaba en los años noventa. Por otro lado, este impulso se manifestó en la cada vez más elevada rentabilidad de

En 2014 se llega a un acuerdo para el pago de la deuda con el Club de París afectada por el default de 2001. También se emite deuda para el pago de la indemnización a Repsol por la nacionalización parcial de Yacimientos Petrolíferos Fiscales (YPF).

Nombre con el que algunos autores se refieren al periodo entre 1976 y 2001, en relación con el funcionamiento de la economía argentina. Véase Basualdo (2006). 
los títulos del BCRA como corolario de la necesidad de la financiación para el pago a acreedores extranjeros y el mantenimiento del tipo de cambio. Al estar en manos de los bancos, los tipos de interés de estos títulos podían influir en los tipos que fijaban para sus servicios.

La utilización prioritaria de recursos para el pago de acreedores llevó a que el mantenimiento de las reservas se volviera imprescindible, para lo que se utilizaron los controles del mercado de divisas (2011) y la reforma de la Carta Orgánica del BCRA (2012).

A pesar de ello, no puede minusvalorarse el hecho de que la deuda pública dejó de desempeñar ese papel como núcleo de la atracción de divisas, no sólo por las condiciones favorables de la cuenta corriente, sino también por las características de la gestión relacionadas con la financiación interna y pública, aunque lo antes expuesto pone ciertas restricciones a la idea de mayor soberanía respecto de los condicionamientos en la política económica y respecto al alcance de la independencia de capitales externos.

El atractivo de la deuda y su lugar prioritario son prueba de que mantiene parte de su papel articulador de la actividad especulativa. No obstante, no es el mismo que antes de la crisis, al dejar de ser el medio principal de ingreso de capitales. A continuación, se verán cuáles han sido los efectos de la regulación financiera en este periodo, cuyas diferencias con la regulación anterior a 2001 podrían tener otras consecuencias en las necesidades de financiación del Estado o, incluso, ser menos beneficiosas para el capital financiero.

\section{LOS EFECTOS DE LA REGULACIÓN FINANCIERA EN EL CAPITAL FINANCIERO ENTRE 2002-2012}

En este apartado analizaremos los efectos de las medidas que afectan al vínculo del capital financiero con el Estado y que en el periodo anterior favorecieron la expansión de las finanzas en Argentina.

El Plan de Convertibilidad generaba la necesidad de ingreso de divisas para mantener la base monetaria a través de una deuda pública cada vez más rentable para atraer capitales a una economía cada vez más inestable. A su vez garantizaba el retorno en dólares a los inversores. La paridad fija y la necesidad de mantener el $100 \%$ de la base monetaria impedían el uso de los instrumentos de política monetaria y cambiaria.

En la posconvertibilidad, el Estado recuperó estos instrumentos e intervino a través de medidas como los subsidios al sector privado y el rescate financiero, esenciales para evitar la debacle del sistema financiero. 
La necesidad de financiación del Estado, el elemento que en el pasado condicionó su vínculo con el capital financiero e hizo protagonistas a las finanzas, aparentó estar "bajo control" durante estos años. No sólo por el fin de la convertibilidad, sino también por la nueva gestión de la deuda pública que, a través de la financiación interna, impidió que se generase la dependencia de entrada de capitales extranjeros a través del endeudamiento.

En el caso de las medidas posconvertibilidad, las políticas monetaria y cambiaria fueron herramientas utilizadas para mejorar la competitividad de los productos argentinos. El mantenimiento del "dólar caro" benefició al sector productivo. En un comienzo, gracias a la devaluación, el encarecimiento de las importaciones resultó en una incipiente recuperación de la industria por sustitución de importaciones. Luego, este efecto fue mermando, aunque permaneció el efecto en las exportaciones, vía abaratamiento de los recursos internos (especialmente de la fuerza de trabajo). Lo que no parece evidente, en todo caso, es que el beneficio al sector productivo significara un perjuicio para las finanzas.

La política de control de cambio y la política de esterilización ${ }^{15}$ beneficiaron al capital financiero, aunque este beneficio haya sido menos evidente.

Se creó un vínculo muy estrecho entre las políticas cambiaria y monetaria, y la financiación intra-sector público para el pago de las obligaciones externas. En este entramado el BCRA ocupaba una posición determinante a través de su intervención y se convirtió en el principal proveedor de recursos al Tesoro. Era la colocación de títulos de esta entidad financiera la que permitía el control del tipo de cambio y la acumulación de reservas internacionales y, a su vez, el pago a los acreedores externos.

Esta acumulación de reservas internacionales se reforzó, de forma indirecta, con el control del mercado de divisas en 2011, cuya intención fue la conservación de sus niveles con este fin. En octubre de 2011, ante una fuga de capitales que llegó a 8.443 millones de dólares, el gobierno decidió implementar una serie de modificaciones normativas que afectaron al plazo máximo para el ingreso de divisas proveniente de las exportaciones e intensificaron los controles de las operaciones en moneda extranjera por parte del BCRA. Los controles se fueron extendiendo hasta que en abril de 2012 se oficializó la suspensión de la posibilidad de comprar moneda extranjera para su "atesoramiento". También afectaron a la obtención de divisas por parte de las empresas extranjeras instaladas en el país para el pago de dividendos a las casas

15

Compra de pesos resultantes de la liquidación de divisas a través de la venta de títulos del Banco Central. 
matrices. A partir de finales de este año se estableció que para cualquier operación en el mercado de cambios que tuviese por objetivo el giro de dividendos al exterior, sería necesaria la autorización previa del BCRA.

Por otro lado, y de forma mucho más directa, la reforma de la Carta Orgánica del Banco Central amplió los márgenes para la utilización de reservas en el pago de obligaciones del Estado y amplió también su financiación a través de adelantos transitorios (se habilitó al BCRA para realizar préstamos al gobierno directamente hasta $12 \%$ de la base monetaria y adelantar fondos no superiores al $10 \%$ de los ingresos fiscales correspondientes al año anterior). Todo ello en detrimento del uso que podría haberse dado de estas reservas en la mejora de las condiciones de vida de la sociedad.

La trama se completa si ańadimos al sector financiero a la cadena de financiación. La mayoría de la deuda del Banco Central se encontraba en manos del sector financiero argentino lo que, de alguna manera, daba continuidad a la financiación del sector público por parte de las entidades financieras, mecanismo presente en la década anterior. Así, la política de esterilización se convirtió, vía colocación de deuda por parte del BCRA en la banca, en uno de los mecanismos que mantenía la financiación que la entidad financiera otorgaba a su vez al Estado Central con el fin de que éste pudiese saldar sus obligaciones.

Asimismo, la deuda generada por el sostenimiento de la política monetaria y cambiaria tenía su propio impacto como instrumento financiero. Su emisión creciente impactaba no sólo en la solvencia del BCRA, sino también en el coste de la misma y, por ende, también en la rentabilidad que se pagaba a los tenedores de esta deuda (entidades financieras).

Como podemos ver en la gráfica 7 , la rentabilidad de los títulos emitidos por el BCRA, calculada como porcentaje de los intereses pagados en el stock de títulos emitidos, ha sido elevada en casi todo el periodo. En 2002 fue muy significativa, llegando casi al 20\%, debido a que se elevaron los tipos con el fin de desincentivar la inversión de capitales en el dólar por sus efectos en el valor del peso. Luego cae, pero en 2005 comienza a crecer hasta alcanzar el 13\% en 2008. No es casual que la rentabilidad comenzará a crecer el mismo ańo en el que se reestructuró la deuda en default. La necesidad de financiación del Estado aumentó en este momento, al retomar los pagos de la deuda en suspensión de pagos y comenzar la gestión que incluye el reemplazo de deuda externa por interna. El aumento de la rentabilidad coincidió con el crecimiento del stock de títulos, respondiendo a la necesidad de hacerse más atractivos.

Una de las razones del cuestionamiento de la política de esterilización fue el efecto que pudo haber tenido la emisión creciente de NOBAC y LEBAC en la solvencia de la entidad. El stock de títulos creció a una tasa promedio de $41 \%$ en 


\section{Marisa Bordón}

Gráfica 7. Rentabilidad de NOBAC y LEBAC, 2002-2012

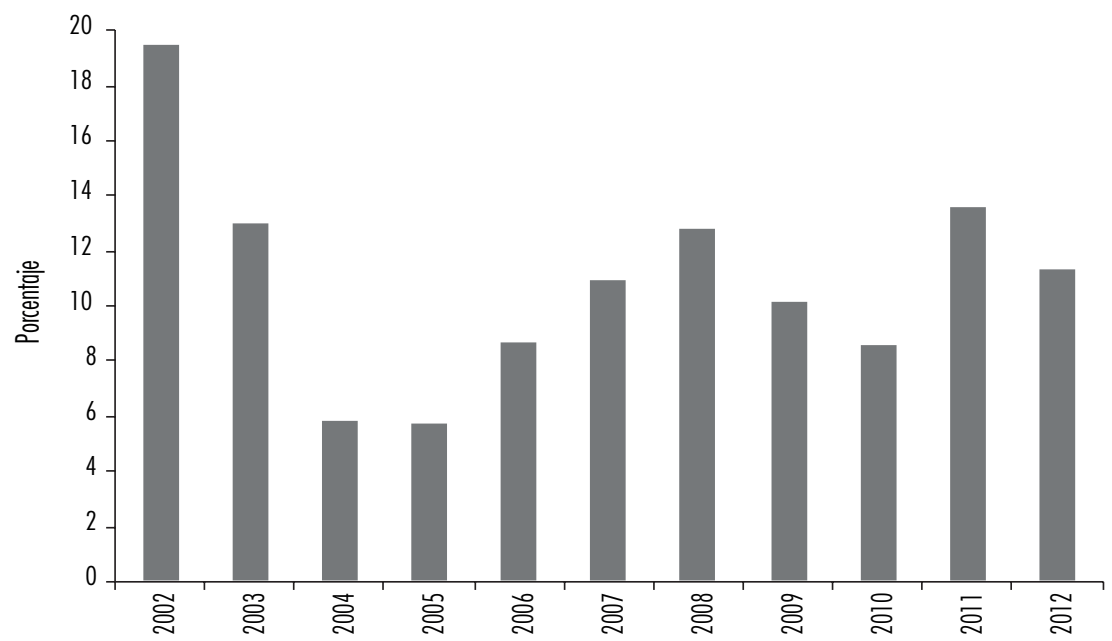

Fuente: elaboración propia con base en datos de los Estados Contables del BCRA de los años 2002 al 2012.

Gráfica 8. Tipos de interés de la deuda argentina* y de la deuda del Tesoro estadounidense, 2002-2012

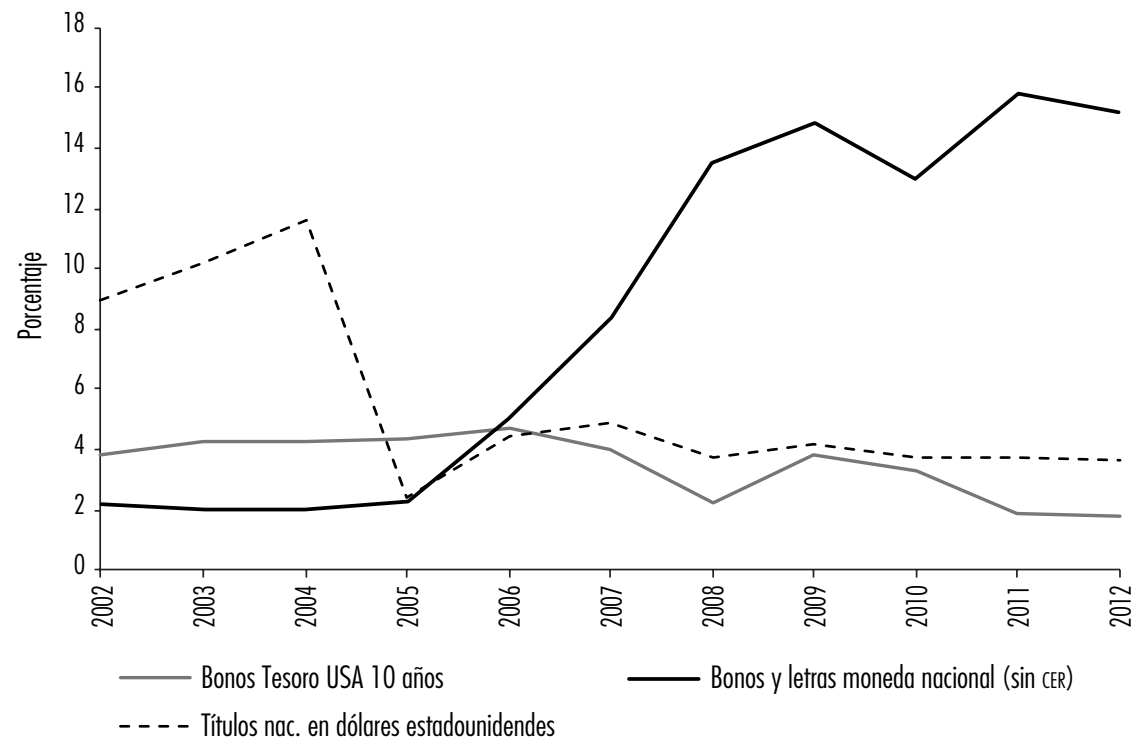

*Los tipos de los bonos argentinos son las tasas promedio ponderadas. El tipo del bono norteamericano a 10 años es el anual publicado el último día del año.

Fuente: elaboración propia con base en datos del Informe de la Deuda de la Secretaría de Finanzas y del Anuario de IAMC 2011 y 2012. 
todo el periodo, pasando de 3.220 a 99.854 millones de pesos (2002-2012). Los intereses pagados por el BCRA, a su vez, crecieron en promedio un $34 \%$ en el mismo periodo, pasando de 626 millones de pesos en 2002 a 13.538 millones de pesos en 2012 según datos presentes en los Estados Contables del BCRA. La deuda del BCRA impacta en el sector financiero, no sólo por representar un elevado beneficio en su haber, sino también por la influencia que los tipos de interés que ofrece han tenido en el resto de las actividades del sector.

Vemos así, que la regulación monetaria y cambiaria favoreció al capital financiero, aseguró el pago de la deuda del Estado y fomentó elevadas rentabilidades en el sector, más allá de haber acabado con la garantía de la paridad un peso un dólar para las inversiones. En este sentido, el ROE del sector financiero siguió una tendencia creciente durante todo el periodo, pasando de $-23 \%$ en 2003 a 11\% en 2007 y al 24\% en 2010 según datos del BCRA. A partir de entonces se convirtió en uno de los más rentables de la economía (más del 25\%). Según Buccieri y Solari (2012, p. 41), el ROE del resto de las actividades presenta una tendencia menos regular con valores similares a los del sector financiero para los ańos 2006 y 2008 (en torno al 15\%), con un máximo del $21 \%$ en 2007, y menores a partir de 2009 (13\% en 2012). ${ }^{16}$

Las políticas del Banco Central, además, permitieron que este modelo macroeconómico del que formaban parte se reprodujera. La acumulación de reservas a que daba lugar permitía sostener este círculo de financiaciones y, además, hacía posible la intervención en el mercado cambiario.

El vínculo entre la financiación del Estado y el endeudamiento prevalece. Si bien el establecimiento de las retenciones a la exportación y el crecimiento de la economía en este periodo fueron fuente de recursos fiscales, en el momento en que esta financiación comienza a presentar limitaciones (2009), se optó por el endeudamiento en lugar de la obtención de recursos, por ejemplo, a través de una reforma fiscal progresiva.

Por otro lado, la reestructuración conservó el privilegio de los acreedores, aunque solventó el problema del pago de las obligaciones a través de nuevo endeudamiento externo. Sin embargo, las elevadas rentas financieras que otorgó la deuda en los años de la posconvertibilidad llevaron a que mantuviera su posición como activo financiero.

16

Los datos de este estudio abarcan 134 empresas de distintos sectores, industria, servicios, construcción, energía eléctrica, y petróleo y gas que cotizan en bolsa, lo que indica que se trata de grandes empresas. 
Los mecanismos de la reestructuración que conservaron la rentabilidad de la deuda han sido canales diferentes a los que representaban los elevados tipos de interés fomentados por la necesidad de financiación y el riesgo país en la década de los noventa No obstante, repercutieron en los costes de la deuda, lo que se hizo patente cuando las cuentas públicas comenzaron a presentar dificultades hasta convertirse en deficitarias a partir de 2009.

Como instrumento financiero la deuda argentina siguió ofreciendo una rentabilidad más elevada que la deuda de los países avanzados. En la gráfica 8 podemos apreciar la diferencia que existe entre los tipos ofrecidos por la deuda nacional y la deuda del Tesoro estadounidense. Si bien los títulos nominados en dólares redujeron sus tipos (incluso fueron menores a los ofrecidos por la deuda estadounidense en 2005, año del canje), se recuperaron a partir de 2006. En el caso de los títulos nominados en moneda nacional no ajustables por inflación, comenzaron ofreciendo unos tipos inferiores a los otros dos, pero en 2006 cambiaron su tendencia. Los tipos ofrecidos por este tipo de activos crecieron aceleradamente de $2.4 \%$ en 2005 a $14.8 \%$ en 2009 y a $15.8 \%$ en 2011.

Gráfica 9. Montos efectivos negociados anualmente de los tres instrumentos con mayor volumen de negociación en millones de pesos, 1990-2012

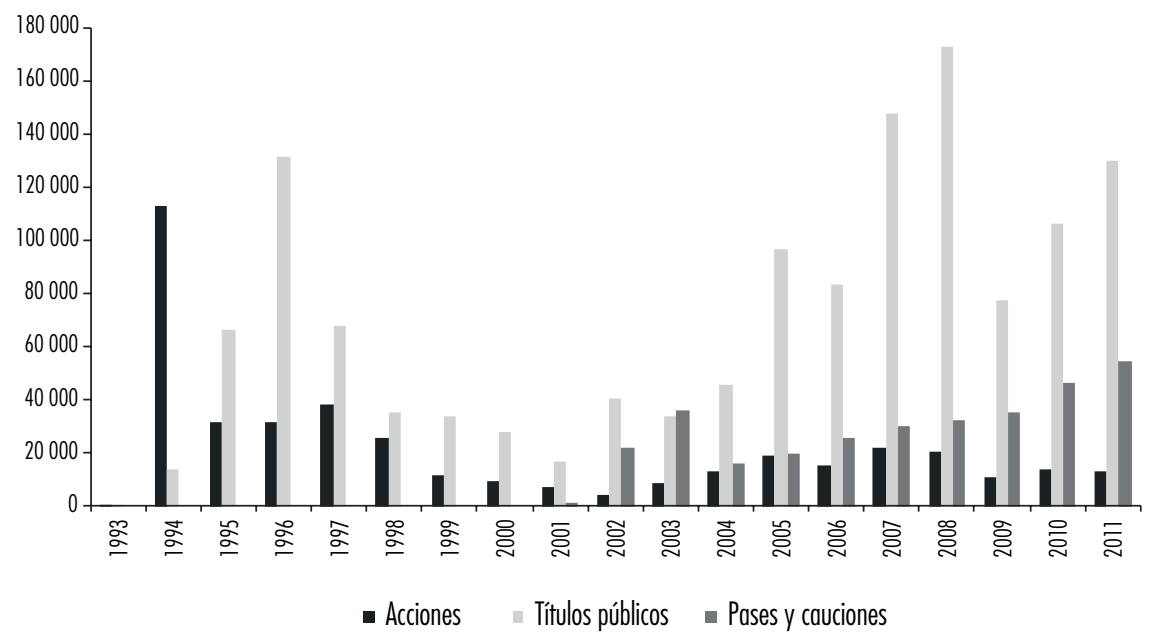

Fuente: elaboración propia con base en datos de la Bolsa de Comercio de Buenos Aires. 
El endeudamiento público mantuvo su importancia como activo financiero. Los títulos públicos siguieron siendo la forma principal en que se instrumentó. La proporción de títulos como componente de la deuda se redujo significativamente durante 2001 y 2004, cuando llegó al 48\%, su nivel más bajo desde 1992. Luego del canje de 2005, esta proporción fue comparativamente más baja que en la década de los noventa, siendo éste uno de los argumentos que se utilizaron para justificar que los mecanismos especulativos del periodo anterior hayan sido desarticulados. No obstante, esta proporción volvió a elevarse en los años siguientes, llegando a niveles similares $(67 \%$ en 2012, mismo porcentaje que en 1995). ${ }^{17}$

Su importancia como activo financiero también se revela en los montos negociados en el mercado de capitales. Han sido los principales instrumentos en cuanto a su monto de negociación anual, como se observa en la gráfica 9. Otra vez vuelve a destacar el año 2005, cuando los montos negociados de títulos públicos se hacen más significativos. En 2007 y 2008 incluso superan los presentes en la década anterior.

En este periodo el mecanismo de la deuda pagada con más deuda que sostiene a la economía en los noventa deja de funcionar. En este sentido la gestión de la deuda y otras medidas que se tomaron en el periodo contribuyen a desactivar este funcionamiento. ${ }^{18}$ No obstante, el alcance de la política económica debe matizarse, en primer lugar, con la presión de la propia inhabilitación de obtener capitales en el exterior después del default y la existencia de elevados precios internacionales de las materias primas para el sostenimiento del modelo macroeconómico. En segundo lugar, la deuda siguió otorgando grandes beneficios a sus tenedores y su pago fue prioridad en tanto que condicionó la política económica del periodo. Lo que nos lleva a un tercer elemento relacionado con este mecanismo; la salida de capitales (la otra cara del mecanismo anterior) continúa (según datos del BCRA, entre 2007 y 2012 se fugan 82.400 millones de dólares) y las medidas aplicadas en este sentido se toman para poder continuar con los pagos de la deuda, no con la intención de romper con el problema estructural de la fuga de capitales. Esto se pone de manifiesto en el momento en el que se toman las medidas (caída de las reservas en 2011,

17

18 
no en el inicio de la fuga en 2007) y en el propio carácter de la medida en forma de autorizaciones del Banco Central, y no a través de ley de capitales extranjeros.

Podemos concluir, a través del análisis del vínculo entre Estado y capital financiero entre 2002 y 2012, que este último conserva su posición central. Esto se explica, en primer lugar, por el mantenimiento parcial del mecanismo de la deuda que conserva su rentabilidad, sus características como activo financiero y el lugar prioritario que mantiene en los objetivos de regulación. En segundo lugar, la regulación financiera no ha cuestionado esta posición, sino que ha contribuido a sostener el mecanismo del endeudamiento y ha conservado ciertos intereses del capital financiero.

\section{CONCLUSIONES}

El discurso oficial presente en nuestro periodo de estudio sostiene que la línea de sus decisiones en materia de política económica, lo que incluiría a las medidas que afectan al ámbito financiero, desarticularon los mecanismos que favorecían al capital financiero vigentes en el periodo de la convertibilidad.

Sin embargo, se verifica que existen elementos que limitan el alcance de esta desarticulación. En primer lugar, la gestión de la deuda no ha implicado un verdadero "desendeudamiento". La reducción del endeudamiento se basa en datos parciales, teniendo en cuenta que tanto el stock de deuda como su coste se han subestimado en los cálculos oficiales de sostenibilidad de la deuda.

Si bien hubo una reducción de su porcentaje en el pIB y de la deuda externa, el alcance de la reestructuración fue limitado en cuanto a su desarticulación como eje de la actividad especulativa. Ha conservado su papel de forma parcial por dos razones. La primera, debido a que la deuda pública conservó su atractivo como canalizador de ahorro externo e interno y continuó siendo el principal activo financiero en los mercados de valores. Además de impulsar indirectamente el crecimiento de las notas y letras del BCRA, instrumentos rentables para los mercados financieros. En segundo lugar, continuó condicionando las políticas económicas y, por tanto, priorizó el desvío de recursos hacia el pago de acreedores frente a destinos alternativos.

La dinámica de endeudamiento interno no liberó de contradicciones al funcionamiento de la economía. Aunque más sostenible que en los años noventa, al evitar la financiación de deuda con más deuda, ha implicado endeudamiento creciente. En este sentido, cobra fuerza la idea de que, en realidad, 
la reestructuración tuvo como objetivo la vuelta a los mercados internacionales de capital y no el fin de la deuda externa y la dependencia que ésta genera.

El alcance de la regulación ha alterado de forma parcial el vínculo entre Estado y capital financiero. La diferencia fundamental ha sido la ruptura de la relación entre la deuda pública y la entrada de capitales internacionales para mantener la política monetaria y el propio pago de la deuda externa ya existente. Por lo demás, el Estado mantuvo las condiciones para la centralidad del capital financiero. La regulación monetaria y cambiaria favoreció al capital financiero, aseguró el pago de la deuda del Estado y fomentó elevadas rentabilidades en el sector financiero. A su vez el capital financiero no perdió por completo su margen de influencia, como prueba de ello los acreedores internacionales "reestructurados" conservaron su posición privilegiada en la lista de prioridades del gobierno.

\section{BIBLIOGRAFÍA}

Administración Nacional de la Seguridad Social (2011-2012), "Fondo de garantía de sustentabilidad del sistema integrado previsional argentino". Informe mensual de Anses (consultado el 1 de diciembre de 2014), disponible en <http://www.fgs.anses.gob.ar/archivos/secciones/Boletin_FGS_11_ 2011.pdf >

Auditoría General de la Nación (2009), "Informe de estudio especial de la deuda pública” (consultado el 25 de julio de 2013), disponible en <http:// www.agn.gov.ar/files/informes/2015_084info.pdf>

Basualdo, E. (2006), Estudios de historia económica argentina: desde mediados del siglo XX a la actualidad, Buenos Aires, Siglo XXI Editores.

Borzel, M. (2005), El manejo de la cuenta capital: enseñanzas recientes y desafíos para Argentina. Documento de trabajo, núm. 7, Buenos Aires, CEFIDAR (Centro de Economía y Finanzas para el Desarrollo de la Argentina).

Buccieri, M. V. \& Solari, A. F. (2012), "Ganancias en Argentina: una perspectiva empírica”, en Ministerio de Economía y Finanzas Públicas, Informe económico trimestral, núm. 80, Buenos Aires, MECON, 19-41.

Capello, M. \& Grión, N. (2010), "Los resultados de la política de desendeudamiento", Monitor Fiscal, año 5, edición núm. 13, IERAL, Fundación Mediterránea, 28 de marzo de 2010.

CENDA (2010), La anatomía del nuevo patrón de crecimiento y la encrucijada actual: La economía argentina en el periodo 2002-2010, Buenos Aires, Atuel. 
Cobe, L. (2009), La salida de la convertibilidad: Los bancos y la pesificación, Buenos Aires, Capital Intelectual.

Damill, M., Frenkel, R. \& Rapetti, M. (2005), "La deuda argentina: Historia, default y reestructuración", en Desarrollo Económico, vol. 45, núm. 178, 187-233, julio-septiembre.

Giuliano, H. L. (2006), Problemática de la deuda pública argentina: La deuda bajo la administración Kirchner, Buenos Aires, Grupo Editor del Encuentro. (2013), "Deuda para todos: la deuda pública nacional”, en Revista Argentina Virtual \& Actual, núm. 55, año 12, mayo-julio (consultado el 23 de julio de 2013), disponible en <http://www.ucalp.edu.ar/sites/default/files/pdf/revista-argentina-virtual-actual/nro55-2013.pdf>

Golla, J. (2006), Dimensión de los conglomerados financieros: el caso argentino. Documento de trabajo, núm. 10, Buenos Aires, Cefidar (Centro de Economía y Finanzas para el Desarrollo de la Argentina).

Golonbek, C. \& Mareso, P. (2011), “Sector financiero argentino 2007-2010: normalización, evolución reciente y principales tendencias". Documento de Trabajo, núm. 36, Buenos Aires, cefidar (Centro de Economía y Finanzas para el Desarrollo de la Argentina).

Kulfas, M. (2014), "La economía argentina, entre la 'década ganada' y los 'fondos buitre', Nueva sociedad, núm. 254, 4-16, noviembre-diciembre.

Kupelian, R. \& Rivas, M. (2011), "Dimensión y regulación de los conglomerados financieros II. El caso argentino", Documento de trabajo, núm. 41,Buenos Aires, Cefidar (Centro de Economía y Finanzas para el Desarrollo de la Argentina).

Lo Vuolo, R. M. \& Seppi, F. (2008), "La falacia del desendeudamiento del sector público argentino", Documento de trabajo 61, Buenos Aires, CIEPP.

Ministerio de Economía y Finanzas Públicas (1993-2012), Boletín Fiscal Trimestral, Buenos Aires, MECon (consultado el 10 de diciembre de 2014), disponible en <http://www.mecon.gov.ar/onp/html/ejectexto/bole_trim_ dosuno.html?var1 =boletrimdossiete>

(2012), "Informe económico trimestral", núm. 80, Buenos Aires, MECON (consultado el 19 de agosto de 2014), disponible en <http://www. economia.gob.ar/peconomica/informe/informe80/version_completa.pdf>

Nemiña, P. (2012), "Argentina: Camino del desendeudamiento (19912011)", Revista Ola Financiera, vol.5, núm. 12, México, unAm, 70-88, mayo-agosto.

Peralta Ramos, M. (2007), La economía política argentina: Poder y clases sociales, 1930-2006, Buenos Aires, Fondo de Cultura Económica. 